\title{
Challenges in modelling the proportion of undiagnosed HIV infections in Sweden
}

Emmi Andersson 1,2, Fumiyo Nakagawa33, Ard van Sighem, Maria Axelsson5, Andrew N Phillips ${ }^{3}$, Anders Sönnerborg ${ }^{1,2,6,7}$, Jan Albert $^{2,8}$

1. Division of Clinical Microbiology, Department of Laboratory Medicine, Karolinska Institute, Stockholm, Sweden

2. Department of Clinical Microbiology, Karolinska University Hospital, Stockholm, Sweden

3. Institute for Global Health, University College London, London, United Kingdom

4. Stichting HIV Monitoring, Amsterdam, the Netherlands

5. Department of Public Health Analysis and Data Management, Public Health Agency of Sweden, Solna, Sweden

6. Department of Infectious Diseases, Karolinska University Hospital, Stockholm, Sweden

7. Unit of Infectious Diseases, Department of Medicine Huddinge, Karolinska Institute, Stockholm, Sweden

8. Department of Microbiology, Tumor and Cell Biology, Karolinska Institute, Stockholm, Sweden

Correspondence: Emmi Andersson (emmi.andersson@ki.se)

Citation style for this article:

Andersson Emmi, Nakagawa Fumiyo, van Sighem Ard, Axelsson Maria, Phillips Andrew N, Sönnerborg Anders, Albert Jan. Challenges in modelling the proportion of undiagnosed HIV infections in Sweden. Euro Surveill. 2019;24(14):pii=1800203. https://doi.org/10.2807/1560-7917.ES.2019.24.14.1800203

Background: Sweden has a low HIV prevalence. However, among new HIV diagnoses in 2016, the proportion of late presenters and migrants was high ( $59 \%$ and $81 \%$, respectively). This poses challenges in estimating the proportion of undiagnosed persons living with HIV (PLHIV). Aim: To estimate the proportion of undiagnosed PLHIV in Sweden comparing two models with different demands on data availability and modelling expertise. Methods: An individual-based stochastic simulation model of HIV positive populations (SSOPHIE) and the incidence method of the European Centre for Disease Prevention and Control (ECDC) HIV Modelling Tool were applied to clinical, surveillance and migration data from Sweden 1980-2016. Results: SSOPHIE estimated that the proportion of undiagnosed PLHIV in 2013 was 26\% ( $n=2,100 ; 90 \%$ plausibility range (PR): 900-5,000) for all PLHIV, 17\% $(n=600$; 90\% PR: 100-2,000) for men who have sex with men (MSM), 35\% in male ( $n=300 ; 90 \%$ PR: $200-700)$ and $34 \%$ in female ( $n=400 ; 90 \%$ PR: $200-800)$ migrants from sub-Saharan Africa (SSA). The estimates for the ECDC model in 2013 were $21 \%(n=2,013 ; 95 \%$ confidence interval (CI): 1,831-2,189) for all PLHIV, $15 \%$ ( $n=369 ; 95 \% \mathrm{Cl}: 299-434)$ for MSM and 21\% ( $n=530$; 95\% Cl: 436-632) for migrants from SSA. Conclusions: The proportion of undiagnosed PLHIV in Sweden is uncertain. SSOPHIE estimates had wide PR. The ECDC model estimates were unreliable because migration was not accounted for. Better migration data and estimation methods are required to obtain reliable estimates of proportions of undiagnosed PLHIV in similar settings.

\section{Introduction}

Estimating the number of undiagnosed persons living with HIV (PLHIV) is crucial to describe the HIV care continuum and to address the Joint United Nations Programme on HIV/AIDS (UNAIDS) 90-90-90 target [1]. This requires reliable data on the number of undiagnosed and diagnosed PLHIV, the proportion of diagnosed PLHIV receiving antiretroviral therapy (ART) and the proportion of those receiving ART who are virally suppressed [1]. Unlike the other parameters in the HIV care continuum, the undiagnosed proportion of PLHIV cannot be directly monitored and must be estimated.

Sweden has a low prevalence of diagnosed PLHIV (0.07\% (data not shown) compared to $0.4 \%$ in adults in the European World Health Organization (WHO) Region [2]) and a low incidence of new HIV diagnoses, with 430 new cases reported within the national surveillance programme in 2016. The number of new cases with reported transmission within Sweden has decreased and has remained below 100 cases per year between 2013 and 2017 [3]. Thus, most HIV infections diagnosed in Sweden are reported to have been acquired abroad. A large proportion of new HIV diagnoses in Sweden occur in migrants with $81 \%$ in 2016 (77\%, 72\%, 80\%, 84\% and 80\% between 2011 and 2015, respectively) according to data from the Public Health Agency of Sweden (data not shown). Migrants from sub-Saharan Africa (SSA) constitute the largest group ( $38 \%$ of all new infections in 2016) and most of them are reported to have acquired their HIV infection in their country of origin. However, a recent study indicates that doctors underestimated the occurrence of transmissions after arrival to Sweden. Thus, a CD4+ T-cell decline trajectory model applied to migrants diagnosed with HIV in Sweden 1983-2013 estimated that $19 \%$ of migrants were infected post-migration, rather than $12 \%$ as officially reported [4]. Furthermore, between 2009 and 2012, 58\% of individuals diagnosed 
TABLE 1

Data items and sources to inform the ECDC HIV Modelling Tool

\begin{tabular}{|c|c|c|c|c|}
\hline Data item (per year) & Data source 1 & Year & Data source 2 & Year \\
\hline Number of HIV-1 diagnoses & $\begin{array}{l}\text { Public Health Agency of } \\
\text { Sweden }\end{array}$ & $1984-1995$ & InfCareHIV & $1996-2016$ \\
\hline Number of AIDS diagnoses & $\begin{array}{l}\text { Public Health Agency of } \\
\text { Sweden }\end{array}$ & $1983-2008$ & InfCareHIV & $2009-2016$ \\
\hline Number of simultaneous HIV/AIDS diagnoses & $\begin{array}{l}\text { Public Health Agency of } \\
\text { Sweden }\end{array}$ & $1983-2002$ & InfCareHIV & $2003-2016$ \\
\hline Number of deaths in HIV-1 infected ${ }^{a}$ & $\begin{array}{l}\text { Public Health Agency of } \\
\text { Sweden }\end{array}$ & $1983-1994$ & InfCareHIV & $1995-2016$ \\
\hline $\begin{array}{l}\text { Number of HIV-1 diagnoses with } \mathrm{CD}_{4}+\geq 500 \mathrm{cells} / \mu \mathrm{l} \text { and no } \\
\text { concurrent AIDS diagnosis }\end{array}$ & InfCareHIV & $1983-2016$ & & \\
\hline $\begin{array}{l}\text { Number of HIV diagnoses with CD4+ 350-499 cells/ } \mu \text { l and no } \\
\text { concurrent AIDS diagnosis }\end{array}$ & InfCareHIV & $1983-2016$ & & \\
\hline $\begin{array}{l}\text { Number of HIV diagnoses with CD4+200-349 cells/ } \mu \text { l and no } \\
\text { concurrent AIDS diagnosis }\end{array}$ & InfCareHIV & $1983-2016$ & & \\
\hline
\end{tabular}

AIDS: acquired immunodeficiency syndrome; ECDC: European Centre for Disease Prevention and Control; InfCareHIV: National Quality Registry for HIV.

${ }^{a}$ For heterosexual migrants from sub-Sahara, data source 1 covered 1988-2006 and data source 2 covered $2007-13$.

with HIV in Sweden were late presenters [5], as defined by a $\mathrm{CD}_{4}+\mathrm{T}$-lymphocyte $\left(\mathrm{CD}_{4}+\right)$ count $<350$ cells $/ \mu \mathrm{l}$ at diagnosis [6]. Late presentation was associated with origin in SSA, eastern Europe, Asia and the Pacific region [5]. Data from the National Quality Registry for HIV (InfCareHIV) [7] Sweden shows that late presentation still is common (59\% in 2016) (data not shown).

In Sweden, most parameters of the HIV care continuum are well documented in InfCareHIV, which has had national coverage since 2008 . Sweden was reported to be the first country to achieve the UNAIDS 90-90-90 target [8] but the estimate of the proportion of undiagnosed PLHIV is uncertain; as in many other European countries [9]. The proportion of undiagnosed PLHIV in Sweden was estimated to be $12-20 \%$ in 2006 by Hamers et al. [10] but no estimates have been published since.

Access to high quality surveillance data combined with near complete coverage of diagnosed individuals with HIV in InfCareHIV since 2008, as well as good coverage of entry of $\mathrm{CD}_{4}+$ cell counts at diagnosis ( $95 \%$ in 2013), country of origin ( $99 \%$ in 2013) and transmission route (96\% in 2013) are good starting points for estimating undiagnosed individuals with HIV in Sweden.

We evaluated two models with different requirements on data availability and modelling expertise to estimate the proportion of undiagnosed PLHIV in Sweden. Migration may bias estimates of undiagnosed HIV infections, because HIV-positive migrants should only be counted as undiagnosed in Sweden after their arrival to the country. The individual based stochastic simulation model of HIV positive populations (SSOPHIE)
[11-13] specifically accounts for both in-country infections and infections likely to have occurred abroad, but it is labour and computing intensive. The incidence method in the HIV Modelling Tool provided by the European Centre for Disease Prevention and Control (ECDC) $[14,15]$ was developed to use routinely collected HIV surveillance data but does not take migration into account. We wanted to compare these available models to see how they performed on data from the Swedish HIV epidemic. Both models produce estimates of several stages of the HIV care continuum but in this analysis, we only focus on estimating the proportion of undiagnosed PLHIV in Sweden.

\section{Methods}

\section{Data sources}

InfCareHIV Sweden is a national quality assurance registry, clinical decision tool and research database. It has full national coverage since 2008 and covers the major cities including Stockholm, Gothenburg and Malmö since the 1980s. Clinical, epidemiological and laboratory data on all registered patients with an HIV diagnosis in Sweden are entered and a well-structured system for quality control ensures data reliability $[4,16]$. As at 1 June 2014 , the date of data extraction used for the SSOPHIE modelling, 9,647 patients were registered in InfCareHIV Sweden. Complementary data extraction for the ECDC Modelling Tool was carried out on 13 March 2017 at which point 10,858 patients were registered. This extraction consisted of data for the years 2014-16 and HIV diagnoses within $\mathrm{CD}_{4}+$ count strata as explained in Table 1. Only accumulated data on patient populations were extracted and no patient identifiers were used. 
TABLE 2

Data items and sources to inform SSOPHIE

\begin{tabular}{|c|c|c|c|c|}
\hline Data item (per year) & Data source 1 & Year & Data source 2 & Year \\
\hline Number of HIV-1 diagnoses ${ }^{a}$ & Public Health Agency of Sweden & $1980-1995$ & InfCareHIV & $1996-2013$ \\
\hline Number of AIDS diagnoses ${ }^{b}$ & Public Health Agency of Sweden & $1980-2008$ & InfCareHIV & $2009-2013$ \\
\hline Number of simultaneous HIV/AIDS diagnoses ${ }^{\mathrm{a}}$ & Public Health Agency of Sweden & $1980-2002$ & InfCareHIV & $2003-2013$ \\
\hline Number of deaths in HIV-1 infected ${ }^{c}$ & Public Health Agency of Sweden & $1983-1994$ & InfCareHIV & $1995-2013$ \\
\hline Median $\mathrm{CD}_{4}+$-count at HIV-1 diagnosis & InfCareHIV & $1983-2013$ & & \\
\hline Number of people seen for HIV-1 care & InfCareHIV & $1983-2013$ & & \\
\hline Number of people receiving ART & InfCareHIV & $1987-2013$ & & \\
\hline $\begin{array}{l}\text { Percent of people diagnosed late }\left(\mathrm{CD}_{4}+<200\right. \\
\text { cells } / \mu \mathrm{l})^{\mathrm{d}}\end{array}$ & InfCareHIV & $1983-2013$ & & \\
\hline $\begin{array}{l}\text { Percent of people diagnosed promptly } \\
{\text { (CD4 }+\geq 350 \text { cells } / \mu \mathrm{l})^{\mathrm{d}}}\end{array}$ & InfCareHIV & $1983-2013$ & & \\
\hline $\begin{array}{l}\text { Percent of people on ART with } C_{4}+\geq 350 \text { cells/ } \\
\mu l^{d}\end{array}$ & InfCareHIV & $1987-2013$ & & \\
\hline $\begin{array}{l}\text { Percent of people on ART with viral load }<500 \\
\text { copies } / \mathrm{ml}\end{array}$ & InfCareHIV & $1996-2013$ & & \\
\hline Death rates in the general population & $\begin{array}{l}\text { The National Board of Health and } \\
\text { Welfare }\end{array}$ & 2008 & & \\
\hline Proportion of HIV-1 infected with HCV/HBV & InfCareHIV & 2014 & & \\
\hline Linkage to care & InfCareHIV & 2013 & & \\
\hline Retention in care & InfCareHIV & 2013 & & \\
\hline Proportion with viral suppression & InfCareHIV & 2013 & & \\
\hline
\end{tabular}

AIDS: acquired immunodeficiency syndrome; ART: antiretroviral therapy; HBV: hepatitis B virus; HCV: hepatitis C virus; InfCareHIV: National Quality Registry for HIV; SSOPHIE: Stochastic Simulation model of Outcomes of People with HIV In Europe.

a Surveillance reports for AIDS from 1980-84 were reregistered after availability of HIV testing in 1985.

b Possible under-reporting after 2005 is compensated by factoring in $15 \%$ in the model.

c For heterosexual migrants from sub-Sahara, data source 1 covered 1988-2006 and data source 2 covered $2007-13$.

${ }^{d} \mathrm{CD}_{4}+$ cell counts in these formats were used only for the whole population.

Since 1985, all HIV cases in Sweden are anonymously reported to the Public Health Agency of Sweden by the clinical doctor and the clinical laboratory that confirmed HIV under the Communicable Diseases Act [17]. From 1983 until 2005 reporting of acquired immunodeficiency syndrome (AIDS) was mandatory. Records of the number of HIV diagnoses, AIDS diagnoses and deaths per year within each transmission group were extracted from 1980 until 2013.

Data on general migration from SSA were used in SSOPHIE. These data were based on the number of permits to stay in Sweden granted to persons born in SSA annually 1980-2014 as recorded by Statistics Sweden ([18] and personal communication Lo Mildh, Statistics Sweden, 30 October 2015). These data were used because both the InfCareHIV database and the Public Health Agency of Sweden have incomplete records regarding when foreign-born HIV-positive persons first arrived in Sweden.

HIV type 2 represents $<0.5 \%$ of all HIV infections in Sweden, due to this we focused on HIV type 1 , hereafter referred to as HIV.

\section{Ethical approval}

Ethical permit 532-11 with amendment T996-11 from the Regional Ethical Review Board in Gothenburg covers this study.

\section{Data items and model populations}

The data used by the models to inform estimates of undiagnosed infection were obtained from InfCareHIV database and the Public Health Agency of Sweden. For each data item, the coverage and data quality for the two sources was assessed over the study period and the source with the best coverage was chosen. For case reporting national surveillance data was used from the beginning of the epidemic until the mid-1990s and InfCareHIV data thereafter. CD4+ cell counts at diagnosis were extracted from InfCareHIV. A slight underreporting of AIDS cases is assumed after 2005 and compensated for by $15 \%$ in SSOPHIE. The data items and their sources are presented in Table 2 for SSOPHIE and Table 1 for the ECDC Modelling Tool.

The continuum of HIV care was monitored using data from the InfCareHIV database; figures on linkage to care, retention in care and retention on ART from 2013 was > $99 \%$, > $99 \%$ and $>98 \%$ respectively. Of patients retained in care, $98.5 \%$ started ART within a year if eligible according to the national treatment guidelines at 
A. HIV incidence

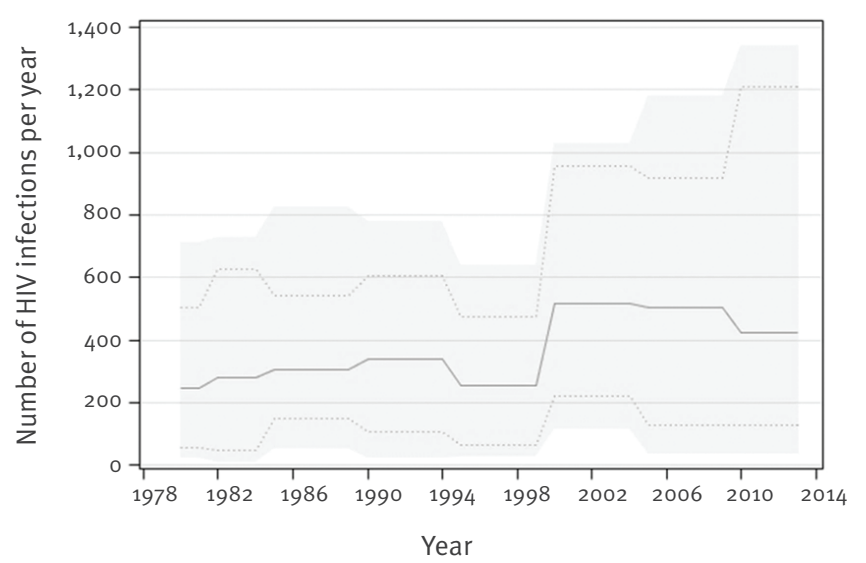

B. HIV diagnosis rate

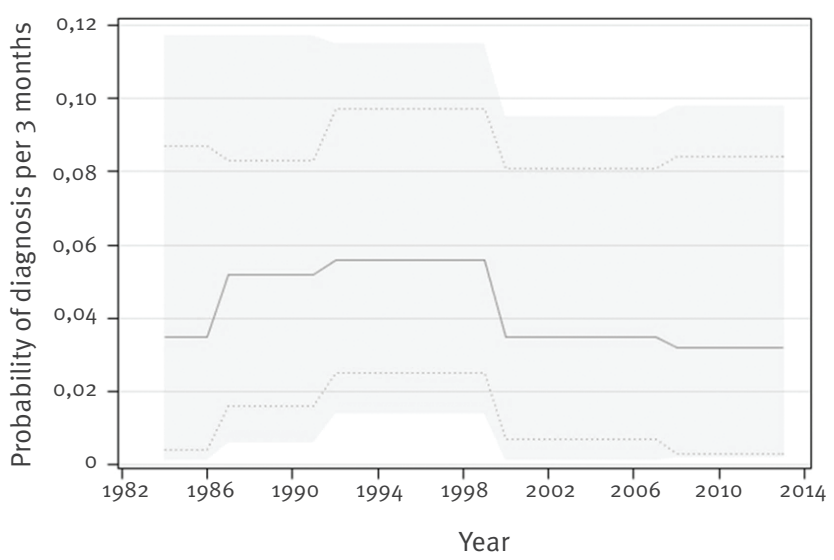

- Median $\quad \cdots . . . . .90 \%$ percentile band $\quad \square$ Max/Min

PLHIV: persons living with HIV.

that time, $94 \%$ of persons on ART had viral loads $<50$ copies/ml (Veronica Svedhem, personal communication, 22 December 2014).

For both models, estimates for the undiagnosed proportion were made for all PLHIV as well as separately for the following transmission groups: men having sex with men (MSM), heterosexually infected migrants from SSA and other heterosexually infected patients, i.e. Swedish-born persons and migrants from other areas in the world than SSA. The number of individuals in the subpopulations do not add up to the total number of PLHIV, because not all PLHIV are part of one of these three subgroups. People with injecting drug use and heterosexually infected migrants from south-east Asia are also relevant transmission groups in Sweden, but they were too small to model individually.

\section{SSOPHIE}

SSOPHIE was developed by Nakagawa et al. within the Stochastic Simulation of Outcomes of People with HIV In Europe project in EuroCoord (EuroCoord-SSOPHIE project) [11-13]. Briefly, the model accounts for domestic and non-domestic infections and uses clinical and observational data to simulate hypothetical HIVinfected individuals from the beginning of a specified HIV epidemic until the present time. Approximate Bayesian computation (ABC) methods are used to calibrate the simulated data to the observed data. The model was developed for settings where extensive surveillance information is available but is flexible with respect to which parameters are required and depending on data reliability, parameters can be weighted and/or fixed. To account for suggested epidemiological differences in access to care, diagnosis rate etc. transmission groups can be categorised in order to calibrate and obtain estimates for the entire population as well as separately for larger subgroups.

Estimates on migration from SSA were included in SSOPHIE. We used the approach described by Nakagawa et al. [13] Briefly, we simulated a 'pool' of HIV-positive people living in SSA using Spectrum/ Estimation and Projection Package (EPP) and an HIVincidence curve based on UNAIDS estimates $[19,20]$. From this pool, we assumed a rate of migration of HIVinfected persons based on the national data on migration from SSA to Sweden, under the assumption that HIV-infected and uninfected persons had a similar rate of migration. This enabled us to make estimates relating to the transmission subgroup of heterosexually infected migrants from SSA.

SSOPHIE was run on data until 2013 for all PLHIV and the subgroups. For the heterosexual transmission groups, estimates were produced separately for men and women. SSOPHIE estimates are given as point estimates with $90 \%$ plausibility ranges (PR) that indicate the certainty of the point estimate.

A total of 30,000 simulations were performed, of which 16,060 were not terminated prematurely; 748 runs were under the calibration-score 0.3 (i.e. they resulted in outputs that were quite close to the observed data with an average deviation from the observed data across all data items $330 \%$ ). We aimed for a 
FIGURE 2

Estimated number of persons living with undiagnosed HIV, by year, Sweden, 1980-2013

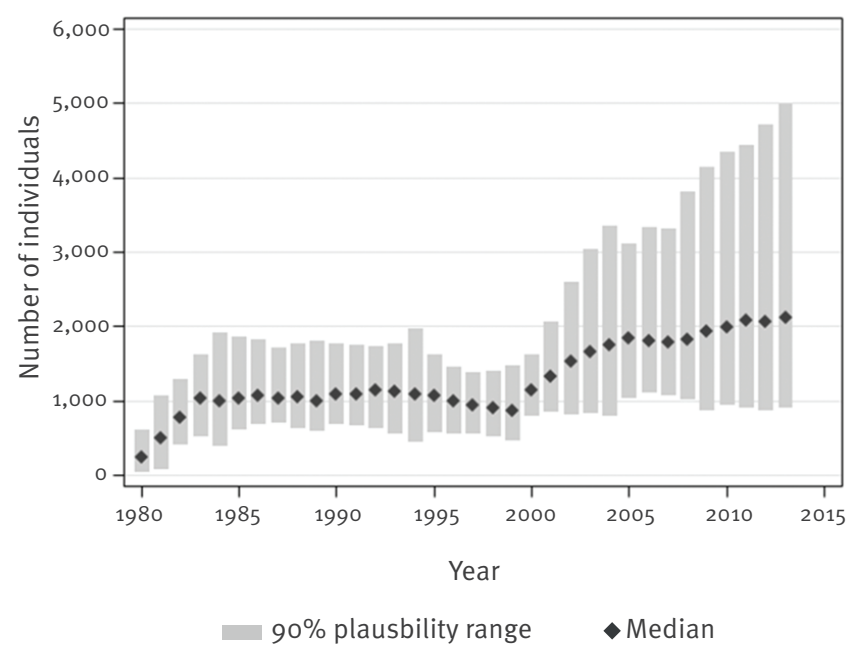

calibration-score as low as possible given that we were attempting to reconcile data from several sources on various aspects of the epidemic. We balanced the aim for a low calibration-score with the desire to have at least 100 parameter sets so that we could consider a range of ways in which the epidemic could be reconstructed. Too few runs (only 17) were under the desired threshold of calibration-score $<0.25$ and the results were therefore based on the 107 parameter sets with calibration-score 0.27 . Data items used for calibration are shown in Supplementary Table S1 and examples in Supplementary Figure S1.

\section{The ECDC HIV Modelling Tool}

The incidence method in the ECDC HIV Modelling Tool version 1.3.0 is a multi-state back-calculation model based on maximum likelihood statistics $[14,15]$. The method first estimates HIV incidence over time and time from infection to diagnosis by $\mathrm{CD}_{4}+$ count strata and then estimates the size of the HIV-positive population. Migration is not addressed in the model but subgroups can be analysed separately.

The model included data from 1983 up to 2016 for all PLHIV and separately for the subgroups MSM, heterosexual migrants from SSA and other heterosexually infected individuals. An optimised model, as assessed by the goodness of fit results, was chosen and the same settings were used for all subgroups. HIV diagnoses were modelled from 1985, but the model was not fitted to data for 1985 and 1986 to avoid the peak of diagnoses reflecting the introduction of HIV-testing. Diagnosis rate was differentiated by $\mathrm{CD}_{4}+$ count categories from 1987 and improvements in fit were obtained by increasing the number of knots in the incidence curve to six and adjusting the length of time intervals (Supplementary Table S2a). Bootstrap analysis (1,000 iterations) was applied to produce $95 \%$ confidence intervals $(\mathrm{Cl})$ of the estimates from the final model.

\section{Results}

\section{SSOPHIE estimates}

Using SSOPHIE, we modelled HIV incidence and HIV diagnosis rate to estimate the proportion of undiagnosed PLHIV. Figure 1a shows that SSOPHIE estimated a median incidence of 400-500 new domestic HIV infections per year during 2000-13 with an increasing $90 \%$ PR. Figure $1 \mathrm{~b}$ shows that the diagnosis rate was estimated to have decreased from the end of the gos until 2013.

The number of undiagnosed individuals among all PLHIV during $1980-2013$ as estimated by SSOPHIE is shown in Figure 2 and the estimates for subgroups are summarised in Table 3. The proportion of undiagnosed PLHIV was $26 \%$ in 2013 ( $n=2,100 ; 90 \%$ PR: $900-5,000)$. The estimate of undiagnosed infections was lowest in MSM; $17 \%(n=600 ; 90 \%$ PR: $100-2,000)$, and highest in heterosexual men from sub-Saharan Africa; $35 \%$ $(n=300 ; 90 \%$ PR: 200-700). The proportion undiagnosed among heterosexually infected persons, excluding migrants from SSA, was $30 \%$ in both men $(n=300$; 90\% PR: $100-1,000)$ and women $(n=400 ; 90 \%$ PR: 100-1,200).

\section{The ECDC HIV Modelling Tool estimates}

The estimates of the HIV incidence and time to diagnosis obtained with the ECDC HIV Modelling Tool are presented in Figure 3. The incidence of HIV infections was estimated to have stabilised between 400 and 500 new infections per year since 2001 (Figure 3a). However, the ECDC model does not account for migration, this means that infection could have occurred abroad and those foreign-born persons could have been living with HIV abroad before being diagnosed in Sweden. The time from infection to diagnosis was estimated to have gradually increased from under 3 years in the 1980 s to 5 years in 2016 (Figure 3b). Time to diagnosis was highest among migrants from SSA, over 6 years since 2011 and lowest in MSM, 3 to 4 years since 2003 (data not shown).

The ECDC model estimates of the proportion of undiagnosed PLHIV in 2013 are shown in Figure 4 and Table 3. The overall estimate was $21 \%(\mathrm{n}=2,013 ; 95 \% \mathrm{Cl}$ : 1,831-2,189), with $15 \%(n=369 ; 95 \% \mathrm{Cl}: 299-434)$ in MSM, $21 \%(\mathrm{n}=530 ; 95 \% \mathrm{Cl}: 436-632)$ in migrants from SSA, and $21 \%(\mathrm{n}=502 ; 95 \% \mathrm{Cl}: 407-600)$ in other heterosexually infected persons. For the ECDC model, input data until 2016 were available and used to obtain estimated proportions of undiagnosed PLHIV (Table 3). The estimate for all PLHIV was $20 \%(n=2,107 ; 95 \% \mathrm{Cl}$ : $1,688-2,577)$, in MSM $17 \%(n=518 ; 95 \% \mathrm{Cl}: 355-706)$, in migrants from SSA $19 \%(n=535 ; 95 \% \mathrm{Cl}: 359-789)$ and in other heterosexually infected persons $22 \%$ $(\mathrm{n}=589 ; 95 \% \mathrm{Cl}$ : 398-884). 
TABLE 3

Proportion and number of undiagnosed PLHIV, as estimated by SSOPHIE and the ECDC HIV Modelling Tool, Sweden, 2013 and 2016

\begin{tabular}{|c|c|c|c|c|c|c|}
\hline \multirow{2}{*}{ Transmission group } & \multicolumn{2}{|c|}{$\begin{array}{c}\text { SSOPHIE } \\
2013\end{array}$} & \multicolumn{2}{|c|}{$\begin{array}{c}\text { ECDC model } \\
2013\end{array}$} & \multicolumn{2}{|c|}{$\begin{array}{c}\text { ECDC model } \\
2016\end{array}$} \\
\hline & Proportion & $\begin{array}{c}n \\
(90 \% \mathrm{PR})\end{array}$ & $\begin{array}{l}\text { Proportion } \\
(95 \% \mathrm{Cl})\end{array}$ & $\begin{array}{c}n \\
(95 \% \mathrm{Cl})\end{array}$ & $\begin{array}{l}\text { Proportion } \\
(95 \% \mathrm{Cl})\end{array}$ & $\begin{array}{c}n \\
(95 \% \mathrm{Cl})\end{array}$ \\
\hline All PLHIVa & $26 \%$ & $2,100(900-5,000)$ & $21 \%(20-23 \%)$ & $\begin{array}{c}2,013 \\
(1,831-2,189) \\
\end{array}$ & $20 \%(17-23 \%)$ & $\begin{array}{c}2,107 \\
(1,688-2,577) \\
\end{array}$ \\
\hline MSM & $17 \%$ & $600(100-2,000)$ & $15 \%(12-17 \%)$ & $369(299-434)$ & $17 \%(13-22 \%)$ & $518(355-706)$ \\
\hline Migrants from SSA & & & $21 \%(18-24 \%)$ & $530(436-632)$ & $19 \%(14-26 \%)$ & $535(359-789)$ \\
\hline Male & $35 \%$ & $300(200-700)$ & & & & \\
\hline Female & $34 \%$ & $400(200-800)$ & & & & \\
\hline Other heterosexual & & & $21 \%(18-25 \%)$ & $502(407-600)$ & $22 \%(16-30 \%)$ & $589(398-884)$ \\
\hline Male & $30 \%$ & $300(100-1,000)$ & & & & \\
\hline Female & $30 \%$ & $400(100-1,200)$ & & & & \\
\hline
\end{tabular}

$\mathrm{Cl}$ : confidence interval; ECDC: European Centre for Disease Prevention and Control; MSM: men having sex with men; PLHIV: persons living with HIV (diagnosed or undiagnosed); PR: plausibility range; SSA: sub-Saharan Africa; SSOPHIE: Stochastic Simulation model of Outcomes of People with HIV In Europe.

a The number of individuals in the subpopulations do not add up to the total number of PLHIV, because not all PLHIV are part of one of these three subgroups.

\section{FIGURE 3}

HIV (A) incidence and (B) time to diagnosis, by year, Sweden, 1980-2016

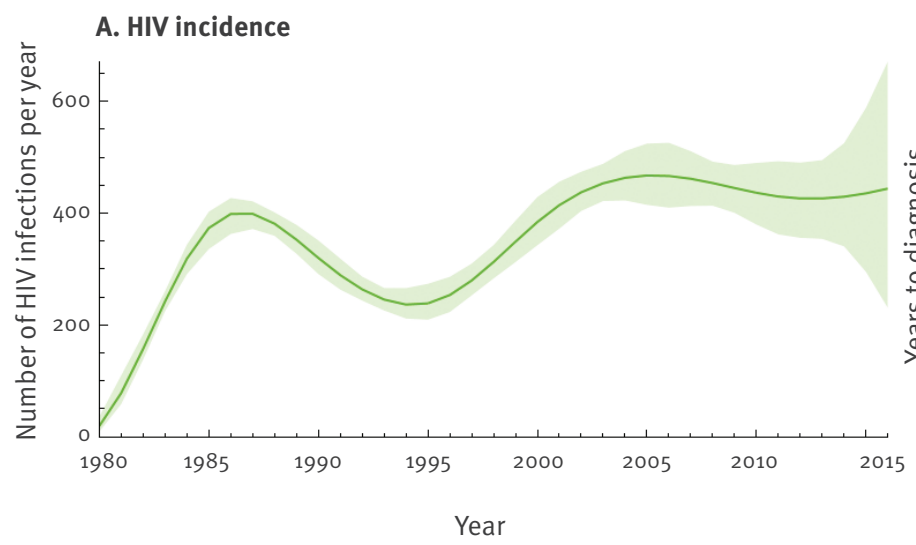

Min-max

\section{Discussion}

We found that the estimated proportion of undiagnosed PLHIV in Sweden in 2013 was comparable between SSOPHIE (26\%) and the ECDC model (21\%). The ECDC model suggested that the proportion of undiagnosed was similar in 2016 (20\%). Both methods estimated around 2,000 individuals living with undiagnosed HIV in Sweden. However, the SSOPHIE estimate had a wide PR and the ECDC model did not account for migration, which makes the accuracy of both estimates uncertain.

The domestic incidence of HIV infections in Sweden estimated with SSOPHIE (400-500 infections per year during 2000-13) seems unrealistically high given that a total of 400-500 new HIV cases (domestic and nondomestic) per year were reported to the Public Health

\section{B. Time to HIV diagnosis}

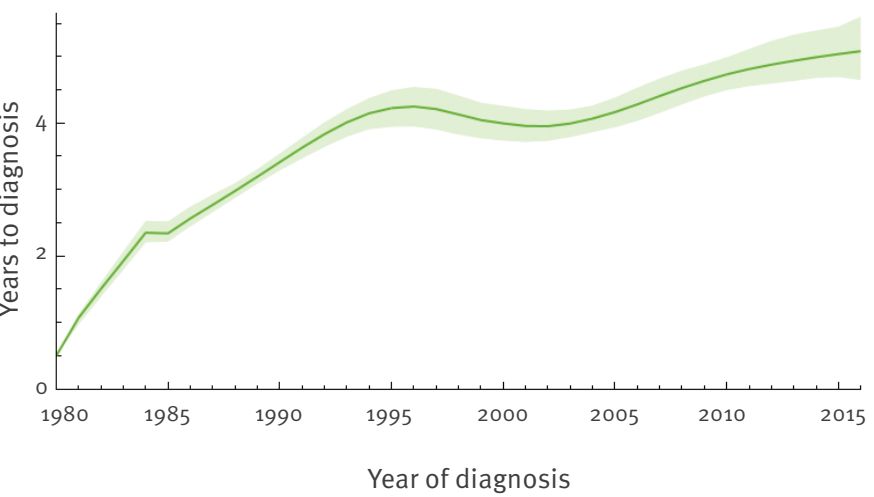

— Mean

Agency of Sweden during the same time period of whom a majority (76\% in 2013) were reported to be non-domestic [3]. The ECDC model had narrow 95\% Cl when considering the wide PR from SSOPHIE, but did not adjust for infections acquired abroad and therefore did not take into account that migrant PLHIV only contribute to the undiagnosed fraction after arrival to Sweden. Consequently, the SSOPHIE and the ECDC point estimates should be interpreted with caution.

Based on the preconception regarding the proportion of undiagnosed PLHIV among Swedish epidemiologists and clinicians, both estimates are higher than expected. The estimates also exceed the $12-20 \%$ undiagnosed in 2006 estimated by Hamers et al. utilising surveillance data from 2005 and the HIV Synthesis 


\section{FIGURE 4}

Number of diagnosed and undiagnosed persons living with HIV, by year, Sweden, 1980-2016

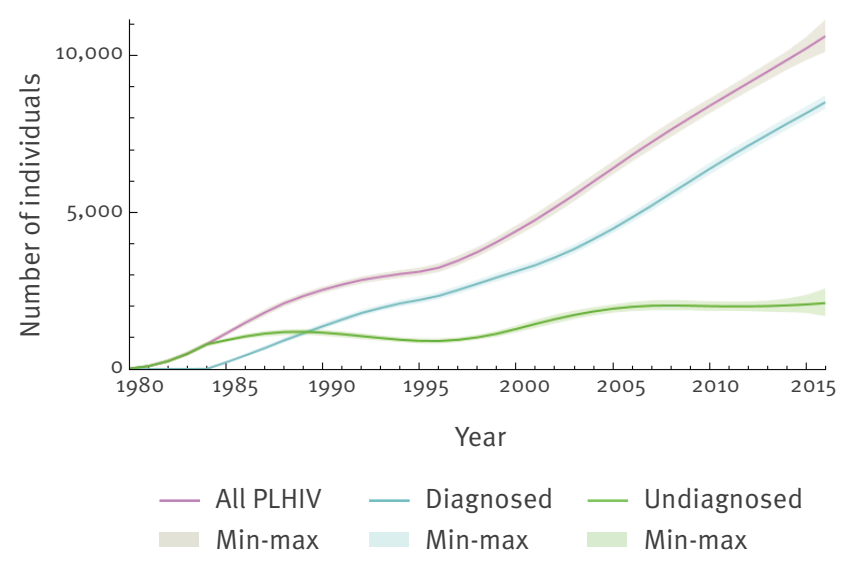

PLHIV: persons living with HIV.

model (a predecessor of SSOPHIE) [10]. In our study, both models estimated around 30\% undiagnosed infections in 2006 (data not shown). Neither are the results in line with an estimate of $10.8 \%$ in 2015 - based on advanced modelling on multiple-biomarker estimates of time between infection and diagnosis [21].

The point estimates for undiagnosed infections in male (35\%) and female (34\%) migrants from SSA and in other individuals with heterosexual transmission (30\%) were higher in SSOPHIE, but the PRs of SSOPHIE overlapped with the $95 \% \mathrm{Cls}$ of the ECDC estimates, indicating that there was no true discrepancy between the results.

Both models predicted that MSM have the lowest proportion of undiagnosed infections. Historically, this subpopulation has been less influenced by migration making it more suitable for modelling using these two methods. However, in 2016, 74\% of MSM diagnosed with HIV in Sweden were reported to have been infected abroad and $71 \%$ were born in another country (data not shown), suggesting that migration is of increasing importance also in this group.

Our surveillance data are of high quality, but had some limitations; CD4+ cell count medians from the 1980s might be slightly biased by incomplete coverage but this is unlikely to have affected our results. The ECDC HIV Modelling Tool takes missing data into account by assuming that $\mathrm{CD}_{4}+$ count data are missing at random in individuals with no concurrent AIDS diagnosis. We expect some under-reporting of AIDS after 2005 when reporting was no longer mandatory. In SSOPHIE, we have compensated for this, but in the ECDC model, missing data on concurrent HIV/AIDS diagnoses could potentially lead to estimates of time from HIV infection that are shorter than the true values.
There are several limitations in estimating the proportion of undiagnosed PLHIV in Sweden using these two models. First, it is difficult to enumerate a 'hidden' population on whom we have no direct data. Second, the models were applied to small numbers with the Swedish HIV epidemic limited to 400-500 new reported cases per year and even smaller numbers for each modelled subgroup. Third, how to properly account for migration, as migrants from SSA and other areas of the world constitute a large proportion of new HIV cases in Sweden. SSOPHIE explicitly attempts to account for migration, but as our migration data were imprecise the HIV prevalence in SSA and the rate of migration from this region to Sweden was used in the model; weaknesses in incomplete migration data from Sweden and any biases introduced by the assumptions on migration of PLHIV from SSA might have affected the results. Also, migration was only considered in the subgroup heterosexually infected migrants from SSA, even though some migrants have other transmission routes and origin. The ECDC model does not account for migration at all. Fourth, the characteristics of the Swedish HIV epidemic have changed substantially since the 1980 s, with migration becoming an increasingly important factor. Thus, the calibration to historical data in both models may not be have been an optimal approach in Sweden. The feasibility of national estimates of undiagnosed infections in a country where both travel and migration have a large impact on the HIV epidemic is dependent on the inclusion of detailed travel and migration data in surveillance reporting as well as the availability of updated and complete national migration data. Modelling approaches for settings with similar patterns of HIV epidemics need to consider migration as an important factor. Since no method will be perfect, comparisons of results from different approaches while understanding their pros and cons are important.

In conclusion, there remains uncertainty over the proportion of undiagnosed PLHIV in Sweden and both methods used have limitations. Consequently, it is difficult to properly evaluate the HIV care continuum in the country. The main challenges with estimating the proportion of undiagnosed PLHIV in Sweden are the high proportion of foreign-born PLHIV, a comparably small number of new cases per year and heterogeneity between subgroups of PLHIV. A combination of better input data on migration and updated or new models are likely required to address these challenges.

\section{Acknowledgements}

The authors acknowledge the use of the UCL Legion High Performance Computing Facility (Legion@UCL), and associated support services, in the completion of this work. We are grateful to Dr Veronica Svedhem for kindly providing data on the continuum of HIV care in Sweden from the InfCareHIV database.

Conflict of interest 
None declared.

\section{Authors' contributions}

EA, JA and AS designed the methodology of data extraction and were responsible for interpretation of the results within the Swedish context. EA performed data extraction from the InfCareHIV database. MA provided surveillance data from Public Health Sweden. FN was responsible for SSOPHIE with support from AP. EA ran the HIV Modelling Tool with support from $A$ van $S$ and MA. EA and JA wrote the manuscript and all authors were responsible for revising the text.

\section{References}

1. World Health Organization (WHO). 90-90-90. An ambitious treatment target to help end the AIDS epidemic. Geneva: WHO. [Accessed 4 Dec 2017]. Available from: http://www.unaids.org/ en/resources/documents/2017/90-90-90

2. World Health Organization (WHO). Global health Observatory data repository. Prevalence of HIV among adults aged 15-49 estimates by WHO region. Geneva: WHO. [Accessed 28 Mar 2019]. Available from: http://apps.who.int/gho/data/view. main.22500WHOREG?lang=en

3. Public Health Agency of Sweden. Sjukdomsstatistik HIV infection. [Statistics on HIV-infection]. Stockholm: Public Health Agency Sweden. [Accessed 1 Apr 2019]. Swedish. Available from: https://www. folkhalsomyndigheten.se/folkhalsorapportering-statistik/ statistikdatabaser-och-visualisering/sjukdomsstatistik/ hivinfektion/

4. Brännström J, Sönnerborg A, Svedhem V, Neogi U, Marrone G. A high rate of HIV-1 acquisition post immigration among migrants in Sweden determined by a CD4 T-cell decline trajectory model. HIV Med. 2017;18(9):677-84. https://doi. org/10.1111/hiv.12509 PMID: 28444865

5. Brännström J, Svedhem Johansson V, Marrone G, Wendah S, Yilmaz A, Blaxhult A, et al. Deficiencies in the health care system contribute to a high rate of late HIV diagnosis in Sweden. HIV Med. 2016;17(6):425-35. https://doi.org/10.1111/ hiv.12321 PMID: 26559921

6. Antinori A, Coenen T, Costagiola D, Dedes N, Ellefson M, Gatell J, et al. Late presentation of HIV infection: a consensus definition. HIV Med. 2011;12(1):61-4. https://doi.org/10.1111/ j.1468-1293.2010.00857.x PMID: 20561080

7. National Quality Registery for HIV (InfCare HIV). English pages. Stockholm: InfCare HIV. [Accessed 28 Mar 2019]. Available from: http://www.kvalitetsregister. se/englishpages/findaregistry/registerarkivenglish/ nationalqualityregistryforhivinfcarehiv.2172.html

8. Gisslén M, Svedhem V, Lindborg L, Flamholc L, Norrgren H, Wendahl S, et al. Sweden, the first country to achieve the Joint United Nations Programme on HIV/AIDS (UNAIDS)/World Health Organization (WHO) 90-90-90 continuum of HIV care targets. HIV Med. 2017;18(4):305-7. https://doi.org/10.1111/hiv.12431 PMID: 27535540

9. Gourlay AJ, Pharris AM, Noori T, Supervie V, Rosinska M, van Sighem A, et al. Towards standardized definitions for monitoring the continuum of HIV care in Europe. AIDS. 2017;31(15):2053-8. https://doi.org/10.1097/ QAD.0000000000001597 PMID: 28906276

10. Hamers FF, Phillips AN. Diagnosed and undiagnosed HIVinfected populations in Europe. HIV Med. 2008;9(s2) Suppl 2;6-12. https://doi.org/10.1111/j.1468-1293.2008.00584.x PMID: 18557863

11. Nakagawa F. Estimation of the size and characteristics of HIV-positive populations in Europe. London: University College London; 2015. Available from: http://discovery.ucl. ac.uk/1471810/

12. Nakagawa F, van Sighem A, Thiebaut $R$, Smith $C$, Ratmann O, Cambiano V, et al. A Method to Estimate the Size and Characteristics of HIV-positive Populations Using an Individual-based Stochastic Simulation Model. Epidemiology. 2016;27(2):247-56. PMID: 26605814

13. Nakagawa FWriting Group on HIV Epidemiologic Estimates in Countries With Migrant Populations From High Prevalence Areas. An epidemiological modelling study to estimate the composition of HIV-positive populations including migrants from endemic settings. AIDS. 2017;31(3):417-25. PMID: 27831947
14. van Sighem A, Nakagawa F, De Angelis D, Quinten C, Bezemer $D$, de Coul EO, et al. Estimating HIV Incidence, Time to Diagnosis, and the Undiagnosed HIV Epidemic Using Routine Surveillance Data. Epidemiology. 2015;26(5):653-60. https:// doi.org/10.1097/EDE.0000000000000324 PMID: 26214334

15. European Centre for Disease Prevention and Control (ECDC). HIV Modelling Tool (software application). Version 1.3.0. Stockholm: ECDC; 2017. Available from: https://ecdc.europa. eu/en/publications-data/hiv-modelling-tool

16. Helleberg M, Häggblom A, Sönnerborg A, Obel N. HIV care in the Swedish-Danish HIV cohort 1995-2010, closing the gaps. PLoS One. 2013;8(8):e72257. https://doi.org/10.1371/journal. pone.0072257 PMID: 23967292

17. Sveriges Riksdag. Smittskyddslag (2004:168). [Infectious Diseases Act (2004:168)]. Stockholm: Sveriges Riksdag; 2004. Swedish. Available from: http://www.riksdagen.se/ sv/dokument-lagar/dokument/svensk-forfattningssamling/ smittskyddslag-2004168_sfs-2004-168

18. Statistikmyndigheten (SCB). Stockholm: SCB. [Accessed 1 Oct 2015]. Swedish. Available from: www.scb.se

19. Brown T, Bao L, Eaton JW, Hogan DR, Mahy M, Marsh K, et al. Improvements in prevalence trend fitting and incidence estimation in EPP 2013. AIDS. 2014;28(Suppl 4):S415-25. https://doi.org/10.1097/QAD.0000000000000454 PMID: 25406747

20. Stover J, Andreev K, Slaymaker E, Gopalappa C, Sabin K, Velasquez C, et al. Updates to the spectrum model to estimate key HIV indicators for adults and children. AIDS. 2014;28(Supp 4):S427-34. https://doi.org/10.1097/QAD.0000000000000483 PMID: 25406748

21. Giardina F, Romero-Severson EO, Axelsson M, Leitner T, Britton T, Albert J. Getting more from heterogeneous HIV-1 surveillance data in a high immigration country: estimation of incidence and undiagnosed population size using multiple biomarkers. Preprint. Posted 17 Jun 2018. bioRxiv.

\section{License, supplementary material and copyright}

This is an open-access article distributed under the terms of the Creative Commons Attribution (CC BY 4.0) Licence. You may share and adapt the material, but must give appropriate credit to the source, provide a link to the licence and indicate if changes were made.

Any supplementary material referenced in the article can be found in the online version.

This article is copyright of the authors or their affiliated institutions, 2019. 